\title{
« DESTRUCTION DE LA LANGUE » DANS LA PREMIÈRE GUVRE DADA : DES IMPERTINENCES LEXICALES À LA THÉÂTRALITÉ
}

\author{
Mariana KunEŠOVÁ \\ Université d'Ostrava
}

\begin{abstract}
En): The paper deals with the issue of signification in Dada creation which is well known for its goal of "abolition of logic", and its literary consequence: structures not respecting the speech acts to the point that they seem to destroy the very possibility of presence of acceptable significations. The author of the paper, suggesting to name these rebel structures «motivation attacks », starts her research from the famous interpretation of Dada activity by Jacques Rivière : "It is impossible for a man to say something that has no meaning » $(\mathrm{La}$ Nouvelle Revue Française, 1920), and puts out these main questions: What can be the concrete impact of motivation attacks? To which acceptable significations can they lead? Can these have a different form than the one of general connotations of chaos or aggression? The corpus of the research is La Première aventure céleste de Monsieur Antipyrine by Tristan Tzara - the legendary first literary Dada opus, being nevertheless little known itself. The choice of the text is due also to its specific genre: written in a theatrical form, this « collage » is likely to have no other characteristics of scenicity and thus to be theatrically nonoperational. However, the signification, precisely, is a potential means capable of ensuring the attention of the public during the performance. Thus, an analysis of signification should also contribute to clarifying the question of the theatrical potential of La Première aventure.
\end{abstract}

Keywords (En): Dada; Signification; Isotopy; Theatre; Tzara; Prosody

Mots-clés (Fr): Dada ; Signification ; Isotopie ; Théâtre ; Tzara ; Prosodie

\section{Remarques préliminaires}

Demandant et pratiquant une «abolition de la logique ", Dada tient à mettre une fin radicale aux schémas traditionnels, qu'il juge scandaleusement périmés. La méthode est notoirement connue : subversion des principes et catégories de base, à commencer par le principe de coopération. D'où un résulat particulièrement aigu au niveau de la parole, célébrissime, signalé par Henri Béhar : la «destruction de la langue $»^{1}$. À l'observer de près, le phénomène équivaut, pragmatiquement parlant, à l'alternance de passages à motivation décodable et de ceux que je propose d'appeler attaques de motivation: des structures qui ne respectent pas les lois du discours au point d'apparaitre comme des courts-circuits isotopiques. D'où le fait que dans les textes Dada, la «clarté », instrument de la logique et de la communication, et partant l'outil par excellence de la société sinon de la civilisation contre laquelle Dada se révolte, vole en éclats.

Cette crise du sens n'instaure pas moins la question des significations opérationnelles. C'est Jacques Rivière qui le premier, dans sa « Reconnaissance à Dada », l'a pertinemment observé : «il est impossible à l'homme de dire quelque

\footnotetext{
${ }^{1}$ Voir BÉHAR Henri (1979), Tristan Tzara ou la spontanéité, in : Le Théâtre dada et surréaliste, Paris, Gallimard, p. 184, 186, 187. L'auteur se sert notamment des formulations suivantes (je souligne) : «[Tzara] a toujours porté en lui ce feu destructeur du langage » (p. 186); «Si on détruit son mode de communication, l'individu se trouve non seulement isolé, mais ne peut même plus se comprendre soi-même [...] » (p. 187). À partir de ces propositions, j'ai créé la formulation nominale dont je me sers.

Echo des études romanes XI/2, 2015 
chose qui n'ait point de sens $»^{2}$. Or qu'en est-il concrètement des possibles significations des attaques de motivation Dada et de leur impact ? Ces significations peuvent-elles amener un effet autre que des connotations générales de chaos ou d'agression?

Les lignes suivantes rechercheront des réponses à ces questions en étudiant un groupe appartenant aux attaques de motivation les plus agressives de La Première aventure céleste de Monsieur Antipyrine de Tristan Tzara. Le corpus sera donc celui de la légendaire première œuvre Dada, peu connue cependant (1916) ${ }^{3}$.

Le choix du texte découle en outre de ses particularités génériques: ce manifeste Dada en prose, entouré de deux macroparties à apparence de poèmes, consiste en tant que tel dans une pluralité de segments répartis parmi plusieurs personnages. Il dispose donc d'une forme dramatique, et a d'ailleurs été représenté, tant sur la scène du cabaret Voltaire dès 1916 que plus tard à Paris. ${ }^{4}$ Dans le domaine du théâtre, qui lorsque mis en scène, nécessite particulièrement une attention immédiate du récepteur - le public, les significations auxquelles s'ouvre le texte représentent un moyen puissant pouvant l'entraîner. La question de la présence d'un réseau isotopique permettant une possibilité de lecture se pose donc dans le cas de La Première aventure de manière essentielle. D'autant plus que la viabilité théâtrale de ce texte a été souvent contestée, ou du moins mise en doute. ${ }^{5}$ Une étude de ses possibles isotopies pourrait donc contribuer à éclaircir cette problématique.

\footnotetext{
${ }^{2}$ Rivière Jacques, « Reconnaissance à Dada », in La Nouvelle Revue Française, n 83, 1920, p. 220.

${ }^{3}$ Le nom du texte qui démarre l'entreprise Dada n'est pas méconnu, tant s'en faut. Il est également aisé de se le procurer, grâce aux Euvres complètes I de Tzara (Paris, Flammarion, 1975 ; désormais OC) ou à Dada est tatou, tout est Dada (Paris, Flammarion, 1996), ouvrages édités par Henri Béhar. Or il n'est pas sans une certaine signification que La Première aventure soit assez peu citée; par exemple elle ne figure dans aucune anthologie consacrée à l'histoire littéraire du $\mathrm{XX}^{\mathrm{e}}$ siècle. Quant à la recherche portant sur ce texte, il me parait que les résultats des études les plus substantielles, par Henri Béhar, Michel Corvin ou Katherine Papachristos, invitent à être employés afin de développer certaines problématiques que ces auteurs ont découvertes, dont les possibles significations opérationnelles du texte. Les études évoquées signalent au sein de La Première aventure une pluralité de micro-sujets, mais malmenés sinon anéantis par la «destruction de la langue ». Je n'ai pas été confrontée, en revanche, à une étude qui interrogerait dans ce texte les court-circuits de signification de manière systématique; que ce soit pour les étudier isolément, pour observer leur interaction avec un possible réseau de significations opérationnelles, ou pour proposer une lecture de ce texte. Voir BÉHAR, Henri, op. cit., p. 183-214 ; CoRvin, Michel (1971), Le théâtre Dada existe-til ? Tzara et Ribemont-Dessaignes ou la problématique d'un théâtre dada, Revue d'histoire du théâtre 79, p. 219 - 320 ; PAPACHRISTOS, Katherine (1999), L'Inscription de l'oral et de l'écrit dans le théâtre de Tristan Tzara, Montréal, Peter Lang.

${ }^{4} \mathrm{I}^{\mathrm{re}}$ Dada-Soirée, le 14 juillet 1916, Zurich ; Manifestation Dada à l'Euvre, le 27 mars 1920.

${ }^{5}$ Les marques de scénicité du texte sont minces : le dialogue, sauf un cas ponctuel à apparence de provocation, paraît en être absent ; les didascalies manquent complètement. La problématicité du genre a été étudiée dans les publications citées dans la note 3 , en étant interprétée comme une absence de théâtralité (M. Corvin, K. Papachristos), voire comme contenant un rapport orateurpublic $(\mathrm{H}$. Béhar) - mais sans que cette brève constatation contenue dans une note en bas de page (p. 183) soit accompagnée d'une analyse. À ma connaissance, aucune des études concernant Tzara n'examine la théâtralité de La Première aventure telle quelle, dont la corporéité des personnages, les entrées des personnages (ou plutôt leurs prises de voix) ou les adresses à l'interlocuteur. Le point n'a pas été fait non plus des propriétés dramatiques du texte.
} 
Quant au groupe concret des attaques de motivation que j'examinerai, force est de constater d'emblée que dans La Première aventure, ces attaques produisant l'incertitude par rapport à une présence de significations opérationnelles naissent d'une pluralité de facteurs. Le texte, d'une longueur approximative de 8 pages, est en effet un gigantesque collage, dans la macrostructure aussi bien que dans les microstructures. Il se caractérise donc par une hétérogénéité de «matériau » très visible, voire agressive : composition au sens général et particulier, dont genres, réseaux thématiques et contextes d'association, style, acceptabilité d'éléments lexicaux, syntaxiques et logiques, ponctuation, graphie, prosodie. Ces plans, constamment, s'interpénètrent, ce qui renforce l'effet de déstabilisation interprétative. Parmi les attaques de motivation les plus radicales, le groupe le plus nombreux consiste dans des impertinences lexicales. C'est à celui-ci que se consacrera la présente étude.

\section{Impertinences lexicales}

Les impertinences lexicales se servent de moyens très variés, pouvant être répartis dans trois sections comme il suit.

\section{Cases vides ${ }^{6}$}

Les cases vides équivalent aux expressions qui sont pour un lecteur francophone indéchiffrables et produisent une cassure isotopique immédiate. Ces courts-circuits sont fort expressifs, ce qui est dû en une mesure importante à leurs propriétés prosodiques. Celles-ci, en effet, opposent les cases vides à la plupart du lexique français employé, en leur attribuant un caractère de matérialité rudimentaire et un rythme quasi sensuel. ${ }^{7}$ Les cases vides font aisément penser aux

\footnotetext{
${ }^{6}$ Ce concept m'a été suggéré par l'étude de Michaël Riffatterre «La métaphore filée dans la poésie surréaliste », Langue française, 3, 1969, pp. 46-60. M. Riffaterre d'expliquer que «case » est un terme employé par la linguistique anglo-saxonne : « (angl. slot) : position qu'un mot ou un groupe de mots occupe dans un contexte ou dans un structure » (p. 48, note 10). Il s'en sert d'abord pour expliquer le principe de la métaphore filée standard, puis, surtout, en étudiant la métaphore filée surréaliste, dont il compare l'arbitraire à la poésie du «nonsense » : «comme Jabberwocky où les cases réservées aux mots pleins sont occupées par des formes dépourvues de sens » (p. 51 ; je souligne).

7 L'effet de «matérialité rudimentaire » et de dépaysement prosodique découle, comme le montreront les exemples utilisés dans le texte principal, d'abord de l'abondance de consonnes à une sonorité renforcée : sonores $(b, g, d)$, nasales $(m, n)$ et liquides $(r, 1)$, qui souvent se combinent (ng, $\mathrm{mg}, \mathrm{bg}$ ). Ensuite, au début des mots ou des syllabes se placent fréquemment des bilabiales (b, m, p). À l'instar des sonores, elles savent renforcer leur effet en se combinant (mb). Enfin, la distribution de voyelles témoigne souvent de la réduction vocalique à la fin des mots, où apparaissent fréquemment les voyelles les plus fermées (i, ou). La conséquence de cette dernière propriété est que l'accent se place naturellement sur la première syllabe.

Ce sont aussi certains éléments du lexique français qui produisent le même effet, souvent atteint par la répétition, dont : «et nous sommes devenus des réverbères / des réverbères / des réverbères / des réverbères / des réverbères / des réverbères / des réverbères / et puis ils s'en allèrent 》 (noter l'insistance sur la vibrante $r$ et le couple de bilabiales $b / p$ ). Ces emplois soulignent la position de force des cases vides, qui en tant que telles, par leur altérité, s'imposent immanquablement à l'attention.
} 
langues africaines; en tout cas elles ont l'allure d'un lexique exotiquement dépaysant. Par exemple :

$$
\begin{aligned}
& \text { « Mataoi Lounda Ngami » (p. 29) } \\
& \text { «Tombo Matapo » (p. 30) } \\
& \text { «Farafamgama Soco Bgaï Affahou ( (p. 29) }
\end{aligned}
$$

Comme on le voit, les cases vides s'organisent en chaînes, ce qui renforce leur effet; de tension d'abord, due à leur opacité, mais à laquelle se superpose vite une détente, vu le dépaysement prosodique qu'elles entraînent. Dans le texte, elles créent tantôt des vers indépendants, tantôt - ce qui est bien plus déroutant - elles pénètrent à l'intérieur d'un vers disposant d'une continuité isotopique, comme dans les exemples suivants :

$$
\begin{aligned}
& \text { «Mataoi Lunda Ngami avec l'empressement [...] (p. 29) } \\
& \text { «La dilatation des volcans Soco Bgaï Affahou » (p. 32) }
\end{aligned}
$$

Notons que ces cases, bien que «vides », créent une suggestive signification connotée - l'énigme. Celle-ci découle également de leurs formes et propriétés prosodiques, rappelant les formules magiques et faisant naître, à des degrés variés, un effet de dramaticité et un climat de mystère. Voici un exemple de l'énigme à une intensité élevée, à apparence d'incantation :

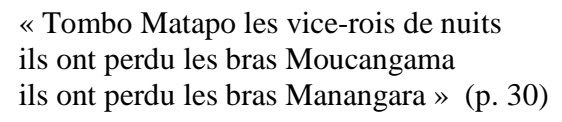

Dans ce passage, c'est le caractère isotopique qui est à remarquer. Davantage : ce «chant», en partie assonancé et presque métrique, est interprétable comme une micro-fable. Ainsi, la dramaticité énigmatique signalée ci-dessus se doit en une mesure considérable justement à ce caractère narratif et formel (le «mètre » et l'assonance œuvrant de sorte que le contenu s'impose à l'attention). À ces propriétés s'allie l'efficacité d'un contenu menaçant, paraissant provenir de la culture des sociétés traditionnelles (des êtres possédant une force surnaturelle qui fatalement perdent leur pouvoir) et une efficacité stylistique, instaurant un même semblant d'origine (cases vides « exoticisantes » et connotant l'énigme; syntaxe rudimentaire, faisant se succéder le sujet et le pronom l'exprimant : " les vicerois... / ils... »). Cette micro-fable à puissance dramatique révèle donc une donne de taille: La Première aventure, où la présence d'isotopies est habituellement signalée comme incertaine, sait s'y ouvrir - et ce à des isotopies perlocutoirement fortes. Parallèlement, ce morceau, comme Dada le souhaitait, casse la stratégie de

\footnotetext{
${ }^{8}$ Les citations proviennent systématiquement de l'édition la plus récente de La Première aventure, in Dada est tatou, tout est Dada, op. cit., contenant par rapport à la version des $O C$ plusieurs rectifications.
} 
la logique discursive, à laquelle le style rappelant les sociétés traditionnelles s'oppose.

Mise à part la connotation d'énigme, les propriétés prosodiques des cases vides tout court sont efficaces, vu leur altérité, également du point de vue auditif, et partant théâtral. Le fait est à nouveau, dans ce texte à apparence scéniquement peu porteuse, essentiel. Le potentiel auditif est particulièrement palpable lors du voisinage des cases vides avec les structures isotopiques, à caractéristiques prosodiques habituelles du français. D'où une rupture auditive, pouvant servir comme un enjeu porteur lors d'une mise en scène, par exemple en performance, ou en voix. Un usage similaire concerne la détente et le plaisir procurés par le rythme et les sonorités "premiers ", pouvant être scéniquement exploités afin d'amener un effet porteur : l'oubli de tout intellectualisme, sinon de toute recherche de sens. Ne sommes-nous pas là au cœur de Dada ?

\section{Interjections et jeux écholaliques}

Les interjections, par leur forme et leurs propriétés prosodiques expressives, ainsi que par leur absence de sens dénoté, sont proches des cases vides. ${ }^{9}$ La parenté avec les cases vides est d'autant plus forte que les chaînes d'interjections contiennent souvent des éléments à formes audacieusement insolites. ${ }^{10}$ Ces interjections «alternatives» renforcent l'effet du dépaysement prosodique et l'intérêt auditif du texte, dont il a déjà été question ci-dessus. ${ }^{11}$

Voici quelques exemples; d'abord des interjections à formes standard, puis alternatives :

a) «taratatatatatatatata » (p. 36)

b) «Hohohohihihioho Bang Bang » (p. 34)

c) «zdranga zdranga zdranga zdranga / di di di di di di di di / zoumbaï zoumbaï zoumbaï zoumbaï / dzi dzi dzi dzi dzi dzi dzi dzi » (p. 31)

d) «dzin aha dzin aha bobobo Tyao oahii hii hii / hébooum / iéha iého » (p. 30)

e) « Iuuuuuuuuupht» (p. 33)

On le voit : les interjections s'imposent immédiatement à l'attention car leurs chaînes s'étalent souvent sur un vers entier ou davantage. En outre, elles sont surabondantes (pour 27 « répliques », 9 chaînes d'interjections) ce qui engendre un effet immédiat d'anarchie.

\footnotetext{
${ }^{9}$ La longueur des interjections est d'une ou de deux syllabes, ce qui donne la possibilité de lectures rythmiques variées, mais toujours opposées au rythme du français. C'est que plutôt que de s'organiser en groupes de souffle, la plupart des interjections maintiennent leurs accents (ce fait est dû entre autres au fait qu'aucune liaison n'est possible).

${ }^{10}$ Ceux-ci sont interprétables, certes, comme des cases vides. Mais vu leur brièveté, leur fréquente répétition au sein d'une chaîne, parallèle aux interjections standard (une variante de cette répétition consiste dans un prolongement spectaculaire d'une seule voyelle), et le fait que ces structures sont pour la plupart entourées d'interjections habituelles, elles apparaissent davantage comme des interjections aussi, inédites.

${ }^{11}$ Théoriquement, les interjections alternatives pourraient posséder un effet d'énigme, à l'instar des cases vides. Or leur apparence d'interjections, doublée du voisinage des interjections standard qui transmettent à ces structures leur symbolisme, battent l'effet d'énigme en retraite.
} 
Malgré leurs formes souvent fantaisistes, les interjections ont des significations pour la plupart immédiatement perceptibles. Il s'agit surtout de la gaieté en des coloris divers et intenses, suggérant souvent l'enfance: gaieté enfantine (l'exemple $a$ ci-dessus), quasi «dansante » $(c)$, joie bruyante $(b)$, joie débridée $(d)$. Rares sont les occurrences d'une autre signification (e semble suggérer un mouvement spectaculaire).

Lorsque les longues chaînes d'interjections succèdent aux structures isotopiques ou bien quand elles s'introduisent à leur intérieur, la continuité isotopique de ces structures s'en voit mise en cause et les significations des interjections se greffent sur celles des éléments isotopiques. Le résultat n'en est cependant pas toujours une détente libératrice. C'est que les contenus des structures isotopiques sont loin de signifier une gaieté insouciante, et les interjections souvent renforcent les effets engendrés par les significations sémantiques. D'où diverses formes de joie plus ou moins agressives.

Parmi celles-ci, une bruyante joie provocatrice :

a) « Nous sommes directeurs de cirque, et sifflons parmi les couvents prostitutions théâtres réalités sentiments restaurants Hohohohihihioho Bang Bang » (p. 34)

Ou bien une ironie efficace, orchestrée par une succession de syllabes à apparence innocemment enfantine, s'aidant de l'assonance et d'un quasi-mètre :

b) «l'idéaliste a tant regardé le soleil que son visage

s'aplatissa ${ }^{12}$

taratatatatatatata » (pp. 35-36)

En outre, l'interférence des interjections avec un passage isotopique peut, à la seule lecture, avoir une valeur ambiguë, indécidable : soit agressive, d'attaque, qualifiable d'humour noir, soit d'un gai rejet de tout sérieux. Il est à noter que les contenus isotopiques concernés dénoncent des situations particulièrement graves dont l'absence, chez l'humanité, des aspects véritablement humains ; ou la menace de la mort, interprétable comme une allusion à la Grande Guerre (dans ce deuxième cas, il faut noter le quasi- «mètre» des deux vers isotopiques les mettant en relief) :

c) «il n'y a pas d'humanité il y a les réverbères et les chiens dzin aha dzin aha bobobo Tyao oahii hii hii héboum / iéha iého » (p. 30)

d) « un grand oiseau en vie

ty a o ty a o ty a o

et quatre beaux fusils

zdranga zdranga zdranga zdranga

di di di di di di di di

zoumbaï zoumbaï zoumbaï zoumbaï

dzi dzi dzi dzi dzi dzi dzi dzi » (p. 31)

\footnotetext{
${ }^{12}$ Selon Henri Béhar, cette forme incorrecte fait partie des quelques fautes de grammaire présentes dans les premires textes de Tzara écrits en français (Tzara était bien de langue maternelle roumaine) ; fautes que le fondateur de Dada se serait par la suite appropriées comme faisant partie de sa «marque ».
} 
Dans les cas comme ceux qui viennent d'être évoqués, libre à la mise en scène ou en voix de choisir soit l'effet d'attaque - un renforcement de la tonalité du malaise donc, soit celui de la libération par rapport à toute perspective de malaise. Toujours est-il que le renforcement d'une tonalité ainsi qu'une succession brusque de deux tonalités contraires (malaise $\rightarrow$ rejet de tout sérieux) dont chacune possède une force perlocutoire de taille, sont des possibilités dramatiquement fortes, pouvant être à la source d'un jeu intéressant avec des effets de tension et de détente. De telles possibilités dramatiques, on l'a vu, concernent non seulement les deux derniers passages observés, mais la totalité des rencontres d'une chaîne d'interjections avec un passage isotopique (dans les cas $a$ et $b$ ci-dessus il s'agissait d'un renforcement de tonalité au moyen d'un comique à effet de joie agressive). Ce dramatisme, en outre, est mis en valeur lors des ponctuelles occurrences de l'assonance et d'un quasi-mètre.

Quant aux tonalités encore, il est à souligner que dans les exemples cités, Tzara se révèle maître tant de l'ironie incisive (exemple $a$ : «l'idéaliste a tant regardé le soleil / que son visage s'aplatissa ») que d'un aphorisme également efficace $(c$ : «il n'y a pas d'humanité il y a les réverbères et les chiens $») .{ }^{13}$ Le texte est donc loin de représenter seulement une rencontre de morceaux à caractère hétéroclite, qu'ils soient énigmatiques, menaçants, provocateurs ou plaisants : il sait mettre en place des scénarios et des observations inédits, dont la justesse de vision les rend particulièrement tranchants.

Creusons à présent la question des significations sémantiques que les interférences interjections / passages isotopiques portent. Ces significations, comme on le sait, se sont révélées d'une force perlocutoire importante. Davantage : plusieurs noyaux interprétables sont présents, comme participant d'un réseau isotopique homogène. Ce réseau a pour sujet Dada, et contient deux thématiques principales. D'abord, il s'agit de l'état de la société et des expériences déclencheurs du mouvement, dans les exemples $b-d$ ci-dessus : «l'idéaliste [...] son visage s'aplatissa », «il n'y a pas d'humanité [...]», « un grand oiseau en vie [...] et quatre beaux fusils ». Ensuite, c'est une partie du programme de la révolte Dada, anarchisante, faisant des membres du mouvement les maîtres de jeu d'un «circus mundi » (exemple $a$ ): «Nous sommes directeurs de cirque, et sifflons parmi les couvents prostitutions théâtres réalités sentiments restaurants ».

Les noyaux isotopiques évoqués sont loin d'apparaître à un endroit isolé du texte : ils concernent les trois parties principales de La Première aventure (le segment central en prose - le manifeste, ainsi que les deux macroparties à

\footnotetext{
${ }^{13}$ Pendant l'aventure Dada, Tzara excelle en aphorismes qui semblent tout à fait en mesure de lui assurer une place dans L'Anthologie de l'humour noir de Breton. À ces nombreux tours de force appartiennent, en outre, un autre des vers initiaux de La Première aventure, «Immense panse pense pense et pense pense », et globalement des observations paradoxales - à apparence absurde, mais fort justes («Dada est un microbe vierge »), tout comme des «vérités » à contenu mystificateur, dénonçant la bêtise humaine, celle du comportament mondain et les limites de la parole («Dada est contre la vie chère », « je me trouve très sympathique », etc.). Pourtant, le nom de Tzara est absent de L'Anthologie bretonienne, sans doute en raison des relations des deux hommes, vite devenues tendues.
} 
apparence de poèmes l'entourant). ${ }^{14} \mathrm{Ce}$ qui donne lieu de supposer l'existence d'une ligne de force isotopique traversant le texte entier. En poursuivant l'examen de ces mêmes passages, il est également possible de constater que si le manifeste consiste dans l'exposition du programme Dada (les «directeurs de cirque »), bien que s'auto-ironisant au moyen des attaques de motivation, les parties poétiques équivalent à la suggestion de l'état d'esprit et des expériences dont Dada naît. ${ }^{15} \mathrm{Ce}$ qui permet d'entrevoir, au sein de ce texte souvent appréhendé comme un simple collage d'alogismes, une composition dramatiquement porteuse.

Les jeux écholaliques, moins nombreux que les interjections (5 occurrences contre 9), en sont proches par leur forme et par leur signification immédiate de franche gaieté. Ainsi que, dans la plupart des cas, par leurs effets de dérision ou de libération par rapport à tout sérieux. Certaines des structures à écholalie, par les significations sémantiques qu'elles créent, prolongent la chaine isotopique signalée par les interjections. Et ce, en usant de stratégies stylistiques et contextuelles diverses, et en représentant des micro-scénarios de plusieurs vers, plus longs que dans le cas des interjections.

Ainsi, les passages à écholalie savent mettre considérablement en valeur les effets et les significations que celle-ci entraîne; dans certains cas davantage que les interjections. À cette position de force contribue aussi le fait que l'écholalie se présente souvent au sein du «dénouement» des scénarios la contenant, comme une apothéose. En outre, l'écholalie crée un matériau prosodique en défaisant des structures sémantiques, de quoi surgit de manière plus directe une interrogation sur le sens. Enfin, les scénarios attribuent à l'écholalie le rôle du chant et / ou du débit en chœur. Ces rôles font encore croître l'intérêt auditif et dramatique des structures concernées. Et puisqu'il s'agit de procédés de théâtre, ces rôles peuvent se voir aisément transposés en un jeu scénique.

Viennent à présent deux cas qui illustrent ces spécificités de l'écholalie. Dans le premier, l'exposé d'une chaîne de "vérités générales » s'en prenant gaiement au «caractère » du bourgeois fait à la fin intervenir, tel un discours direct écholalique, le personnage-émetteur. Le passage se transforme de ce fait en un bref monologue chanté :

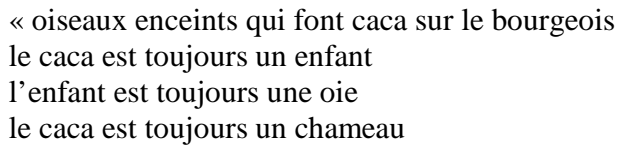

\footnotetext{
${ }^{14}$ Le manifeste contient l'exemple $a$, la première macropartie poétique, les exemples $c, d$, la seconde, l'exemple $b$.

${ }^{15}$ À la stratégie de suggestion du climat dont Dada surgit semble correspondre aussi, bien qu'usant d'un contexte moins proche, le passage isotopique interrogé dans la section «Cases vides »: la micro-fable évoquant des personnages à force surnaturelle qui, fatalement, perdent leur puissance («[...] les vice-rois des nuits »; voir p. 86). N'est-ce pas là une apocalypse, au même titre que la Grande Guerre ? Pour plus d'exemples confirmant la distribution suggestion / exposition, voir la section suivante de cette étude : «Cases pleines », se reporter éventuellement à ma thèse, L'absurde dans le théâtre français Dada et présurréaliste, dirigée par Petr Kyloušek et Didier Plassard, Brno Montpellier 2014.
} 
l'enfant est toujours une oie

et nous chantons

oi oi oi oi oi oi oi oi oi oi oi oi oi oi oi oi oi oi » (p. 32)

Ce morceau est en outre interprétable comme s'inscrivant, bien qu'implicitement, dans la thématique du programme Dada. Par l'attaque du bourgeois et des habitudes contemporaines jugées stériles d'une part. Mais aussi, d'autre part, vu ses choix esthétiques: le plaisir des éléments bannis des esthétiques traditionnelles car bassement matériels (dont « caca »; « oie »), auquel s'ajoute l'effacement des différences au sein d'une pluralité d' «objets» considérés comme violemment incompatibles («caca», «enfant», «oie», « chameau »). D'où une suite de vers à apparence d'un jeu d'enfant, mettant en place un chaos ludique et transgressant toute limite esthétique ou logique. Ce style enfantin, comme il en allait, dans la section «Énigme», pour celui rappelant les sociétés premières, s'oppose à la logique discursive et confirme ainsi les positions Dada.

Le deuxième scénario à écholalie qui mérite l'attention est également interprétable comme exposant l'esthétique Dada. Un élément essentiel de cette esthétique précisément, car attribuant à la «farce»-donc à un «gros » comique, accueillant l'incohérent - la valeur d'un «élément poétique ». À nouveau, l'écholalie, qui se combine ici à une activité néologisante, intervient à la fin de la structure, tel un «chant» en chœur. Qui plus est, ce chant équivaut à une « démonstration pratique » du programme artistique évoqué :

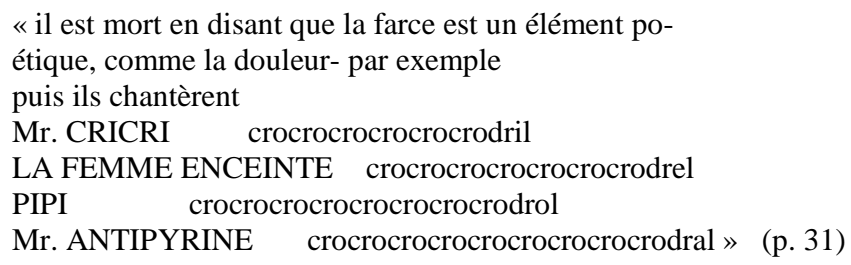

\section{Cases pleines ${ }^{16}$}

Il s'agit d'expressions à sens sémantique, d'emprunts aux champs spécifiques voire spécialisés, insérés au sein des vers: chiffres, termes et formules de géométrie, de chimie et de biologie, comme «fièvre puerpérale », "sangsue », « staphylin », « $\mathrm{H}_{2} \mathrm{O}$ ». Parfois apparaissent des formules et des termes inexistants, mystificateurs ; c'est le cas de deux des trois formules chimiques présentes dans le texte : $\mathrm{SO}_{2} \mathrm{H}_{4} ; \mathrm{Ca}_{2} \mathrm{O}_{4} \mathrm{SPh}$.

Voici quelques exemples de structures à cases pleines :

« la fièvre puerpérale dentelles et $\mathrm{SO}_{2} \mathrm{H}_{4}$ ( (p. 30)

« gonflent les coussins des oiseaux $\mathrm{Ca}_{2} \mathrm{O}_{4} \mathrm{SPh}$ ( (p. 32)

«les robes 7 des anges » $(\text { p. } 32)^{17}$

\footnotetext{
${ }^{16}$ Concept créé par moi-même, inspiré de celui de la case vide (voir la note 6).
} 
De nombreuses cases pleines donc, similairement aux cases vides, consistent dans des matériaux très spécifiques, et la plupart d'entre elles entraînent à ce titre un court-circuit isotopique immédiat et particulièrement agressif. Tel est le cas des formules scientifiques et des chiffres. Dans les cases pleines restantes, représentées non par des formules mais par des mots (termes de biologie et de médecine) - qui donnent le plus l'illusion d'une possibilité d'intersection sémique - le constat de la cassure isotopique se présente parfois après une importante phase d'incertitude dans l'interprétation. À laquelle le contexte peut attribuer le caractère d'énigme, notamment si sont présentes les cases vides, avec leur suggestivité :

«pénètre le désert

creuse le chemin dans le sable gluant

écoute la vibration

la sangsue et le staphylin ${ }^{18}$

Mataoi Lunda Ngami avec l'empressement d'un

enfant qui se tue $\gg($ p. 29)

L'incertitude d'interprétation et l'impression d'un chaos interprétatif sont aussi l'effet qui survient suite au constat des cassures isotopiques : que veulent dire ces passages? Veulent-ils dire quelque chose? Ces interrogations peuvent être accompagnées de la connotation d'une faillite de la logique voire de celle d'un désordre intellectuel. C'est que les cases pleines, à la différence des cases vides et de nombreuses interjections, portent un sens immédiat, et davantage, proviennent des champs de la science. Elles sont donc intuitivement perçues comme garantes d'ordre et de clarté ; valeurs que, cependant, elles malmènent royalement.

Parallèlement aux autres impertinences lexicales, les cases pleines, lorsqu'elles interfèrent avec les structures isotopiques, mettent en circulation des connotations opérationnelles. Puisque les cases pleines possèdent toutes un sens immédiat, ces connotations peuvent naître même lors d'une interférence avec, simplement, d'autres éléments lexicaux. Vu les significations des cases pleines en tant que telles, les connotations issues de ces interférences sont perlocutoirement intenses : elles ont toutes pour noyau, bien que concernant des thématiques diverses, un intense conflit, pouvant se lire comme existentiel. De ce fait, elles s'engagent, et ce de manière importante, dans le réseau isotopique des expériences et de l'atmosphère nourrissant la naissance de Dada. Deux exemples suivent.

\section{Interférence avec une structure isotopique}

Une case pleine dénotant une formule géométrique participe d'une séquence qui est, malgré la présence d'autres attaques de motivation encore (cases vides),

\footnotetext{
${ }^{17}$ On voit que les chiffres, qui semblent pouvoir le plus facilement s'intégrer au sein d'une chaîne verbale, sans produire de cassure isotopique, apparaissent à des positions anarchiques qui leur attribuent la fonction d'impertinences lexicales. Ainsi, un chiffre interrompt le développement d'un syntagme nominal, ou bien il accompagne un référent dont il n'est pas possible d'envisager une pluralité d'exemplaires. Ou encore - ce qui est une des violations les plus brutales du texte - un chiffre remplace la proposition principale d'une phrase complexe.

${ }^{18}$ Dans la présente section, j'effectuerai dans la plupart des citations des soulignements, en me servant systématiquement de caractères gras, voire de l'alternance gras / italique.
} 
interprétable comme isotopique - comme l'allégorie de la destruction de la civilisation, à l'apparence d'une fatalité monstrueuse. Cette structure a déjà été en partie observée dans la section «Cases vides »; la voici à présent dans une forme plus complète :

\author{
« Tombo Matapo les vice-rois de nuits \\ ils ont perdu les bras Moucangama \\ ils ont perdu les bras Manangara \\ ils ont perdu les bras polygone irrégulier \\ à Matzacas la coccinelle est plus grosse que 1'hémi- \\ sphère cérébral » (p. 30)
}

Dans le contexte évoqué, "polygone irrégulier» paraît signifier qu'un des orgueils du monde Belle-Époque, la science, ne s'engage pas à sauver la civilisation, mais est témoin de sa perte. La signification de la faillite de la science découle en une mesure importante de la confrontation de cette formule géométrique avec le style des trois premiers vers, rappelant les sociétés traditionnelles ${ }^{19}$, observé déjà ci-dessus. Un style, donc, rejetant la logique discursive, et par conséquent un monde posant cette logique comme son instrument essentiel.

\title{
Interférence avec une chaîne nominale à signification incertaine
}

Dans une succession d'éléments nominaux ( 2 vers), les cases pleines représentées par des chiffres cassent à trois reprises la possibilité d'isotopies immédiates et entraînent un chaos interprétatif. Ce désordre met cependant intensément en valeur les connotations des éléments de la chaîne nominale : d'une part une pureté céleste, de l'autre, souffrance et mort. Ainsi, les vers discontinus proposent une signification au niveau de la structure profonde ${ }^{20}-$ l'éternel conflit des aspirations de l'homme (pureté) et de son existence terrestre (souffrance, mort) :

\footnotetext{
« aux robes 16 blessés les robes 7 des anges en arc-en-ciel cendre 4 » (p. 32)
}

On le sait, le conflit des aspirations pures et d'une existence terrestre « sale » est un lieu commun souvent répété. Or ici, ces significations apparaissent avec une force perlocutoire élevée. Ce n'est pas seulement le contexte du chaos interprétatif qui, insolite, «nettoie » le potentiel perlocutoire des connotations; ce contexte se

\footnotetext{
${ }^{19}$ L'emplacement de la case pleine est identique à celui des cases vides qui la précèdent. Ainsi, les cases vides transmettent, par parallélisme, leur suggestif effet d'incantation à la case pleine.

${ }^{20}$ Le concept de la structure profonde, issu de la grammaire générative, est fréquemment employé dans l'analyse de théâtre par exemple par Patrice Pavis, au sens d'une acceptabilité au-delà d'une signification immédiate. Voir, entre autres, PAVIS Patrice (2004), Le Théâtre contemporain. Analyse de textes de Sarraute à Vinaver, Paris, Armand Colin.
} 
voit aidé également du dépaysement prosodique ${ }^{21}$. Ainsi, les connotations apparaissent avec une intensité hors du commun.

\section{Vers une conclusion}

Se consacrant aux impertinences lexicales, un groupe particulièrement agressif des attaques de motivation de La Première aventure céleste de Monsieur Antipyrine, les lignes précédentes ont fait apparaître que ces impertinences - cases vides, cases pleines, interjections et structures écholaliques anarchisantes - sont un jeu virtuose des possibilités du mot et de la chaîne verbale, exploitant tant leur côté « matière » que leur sémantisme, et usant de stratégies visant l'intellect aussi bien que de celles fondées sur une suggestivité intuitive.

La caractéristique concernant la signification des structures à impertinences lexicales est de prime abord - nulle surprise puisqu'il s'agit de Tzara - «le Doute » : une intense incertitude d'interprétation, pouvant surgir tant de manière ponctuelle que s'étaler sur des structures prolongées. Or malgré les courts-circuits isotopiques immédiats, ces impertinences peuvent produire des connotations opérationnelles au niveau de la structure profonde, et ce de trois types : énigme, joie et conflit. Ces significations, à tonalités opposées et complémentaires, sont en elles-mêmes perlocutoirement puissantes. Potentiel qui peut considérablement s'accroître d'abord par l'emploi d'effets prosodiques: du «dépaysement prosodique » surtout, mettant en place un rythme et des sonorités « exotiques » et entraînants, à un impact auditif et donc théâtral non négligeable. Parallèlement, certaines structures contiennent un quasi-mètre et parfois, à la fin des vers, une circularité mélodique - c'est-à-dire des régularités qui sont par rapport au contexte exceptionnelles, et imposent les structures concernées à l'attention.

Ensuite, la force perlocutoire des connotations mises en place augmente royalement lors de leur interférence avec les passages isotopiques, d'où une diversité de contextes et de tonalités concrets. Ainsi naissent, malgré une forte diversité contextuelle, des significations lisibles comme formant un réseau de significations, dans lequel se distinguent deux thématiques essentielles : d'une part les expériences et l'état d'esprit qui ont fait naître Dada, de l'autre, le programme Dada. Ces thématiques mettent en scène, entre autres, l'inauthenticité de la société

\footnotetext{
${ }^{21}$ En effet, les cases pleines peuvent aussi héberger des qualités prosodiques. Ces cas ne sont pas systématiques comme dans les deux sections précédentes, mais ponctuels (par exemple les formules scientifiques en font abstraction). Pour la plupart, les qualités prosodiques des cases pleines sont discrètes : ces cases ne créent pas un dépaysement à elles seules. Cependant, elles produisent, dans les vers où elles apparaissent, des mises en valeur des possibilités prosodiques (rythme et $/$ ou allitération). D'où des effets de dramatisation, ou au contraire d'une détente agréablement dépaysante. Exemple d'un effet de dramatisation (allitérations connotant menace) : "écoute la vibration / la sangsue et le staphylin » (p. 29; je souligne). Exemple de dépaysement (rythme et allitérations) : le deuxième vers de la séquence qui vient d'être étudiée, faisant interférer des cases pleines avec une chaîne nominale: «en arc en ciel cendre 4 [quatre] » (p. 32). Les lectures rythmiques de ce dernier vers qui me semblent les plus naturelles sont au nombre de deux :

- «En arc en / ciel / cendre / quatre » (la deuxième moitié du vers fait apparaître un schéma prosodique parallèle)

- «En arc en / ciel / cendre / quat' » (symétrie rythmique entre le deuxième et le quatrième segment).
} 
contemporaine dont le bourgeois et «l'idéaliste»; la menace de la Grande Guerre; une image apocalyptique de la destruction de la civilisation dont la science apparaît comme un témoin impuissant ; l'éternel conflit de la pureté des aspirations de l'homme et de la réalité crue de son existence ; la «farce » en tant que partie intégrante de l'art; le monde appréhendé comme un cirque dont les représentants de Dada se veulent les maîtres.

Ainsi, au niveau de la structure profonde, le texte se montre comme bien plus isotopique qu'on n'aurait tendance à estimer a priori. La ligne isotopique, d'ailleurs, traverse le texte entier, et ce d'une manière compositionnellement efficace : en donnant au segment central, en prose - le manifeste, la valeur de l'exposition d'un programme Dada, et aux macroparties poétiques l'entourant, celles de la suggestion de l'état d'esprit dont Dada découle.

En outre, même les isotopies opérationnelles observables au niveau de la surface sont d'une taille non négligeable. Ces passages savent former des microfables ou scénarios de plusieurs vers (ou lignes, dans la partie en prose), dont l'efficacité dramatique se voit doublée de l'intervention de procédés thêâtraux comme un monologue chanté ou le chant en chœur, invitant à des prolongements scéniques.

Quant aux tonalités concrètes, celles-ci représentent un éventail étonnamment large :

- mystère dépaysant, rejet de tout sérieux et libération par rapport au moindre intellectualisme ou la moindre recherche de sens, souvent accompagnés d'un intense dépaysement prosodique ;

- aphorisme et comique de caractères férocement efficaces ; joie bruyante, agressive ; humour noir ;

- conflit intense, d'ordre existentiel.

Ce sont là des propriétés dramatiques par excellence puissantes, sachant alterner et faire crôtre les effets de tension et de détente. Ce qui peut encore s'intensifier lorsque les tonalités se superposent brusquement, ou que les passages concernés emploient des stratégies stylistiques spécifiques rejetant la logique discursive (style rappelant les sociétés traditionnelles ou celui des comptines enfantines).

Les tonalités, les significations ponctuelles et les courts-circuits isotopiques forment ensemble un contexte de surface à proprement parler inédit, qui entraîne un « recyclage » du lieu commun, afin de le faire intervenir avec sa force initiale, hors du commun. $\mathrm{Vu}$ ce contexte, les significations mises en place tout court ont systématiquement une force perlocutoire élevée. Ainsi, les images à malaise, qui sinon risqueraient l'ennui du lieu commun, sont particulièrement troublantes, les significations ironiques gagnent en force d'incision et les éléments du programme Dada non seulement « épatent », mais s'imposent à l'attention.

En somme, l'étude des impertinences lexicales de La Première aventure montre que ce texte, bien qu'usant de stratégies par excellence Dada, possède un intérêt isotopique de taille, doublé d'un potentiel compositionnel, auditif et dramatique considérable, pouvant se voir aisément prolongé par un jeu scénique. Ces caractéristiques donc, présentent ce texte comme tout à fait convenable pour une mise en scène, en voix ou en performance. 
Certes, il est possible d'objecter que les lectures de certains passages proposées par la présente étude sont dans une certaine mesure virtuelles, ou que plusieurs étapes lors du décodage des structures à attaques de motivation peuvent ne pas concerner tout lecteur / spectateur, car conditionnées par un effort d'interprétation prolongé. Effort dont le lecteur, et encore moins le spectateur, happé par le déroulement du texte dans le temps, peuvent ne pas fournir. Or rappelons qu'œuvrer de sorte que des phénomènes virtuels deviennent matériellement observables est un objectif majeur de la mise en scène, laquelle dispose à ce titre de tout un attirail d'instruments: commençant par des phénomènes prosodiques, et s'ouvrant à une diversité d'effets scéniques et au jeu de l'acteur. Ainsi, bien qu'un spectateur potentiel de La Première aventure ne puisse sans doute pas produire, lors de sa «consommation » du texte, une analyse approfondie, ce texte dispose de propriétés telles que si allié à une mise en scène attentive, il est à même de procurer de manière intuitive la richesse de significations et de tonalités que cette étude a signalées. Telle a d'ailleurs été l'expérience de spectateur de Henri Béhar, confiée lors d'un colloque tenu à propos des avant-gardes voici quelques années : l'expérience d'un spectacle où le texte «tient ${ }^{22}$

Soit dit enfin que les possibilités scéniques de La Première aventure pourraient être creusées au moyen de l'analyse d'autres stratégies la concernant, dont motifs, personnages ou possibilité de dialogues, stratégies auxquelles recourent également d'autres textes dramatiques de Tzara tel le Cőur à gaz. J'espère pouvoir me pencher sur ces questions dans mes travaux futurs.

\section{BIBLIOGRAPHIE}

BÉHAR Henri (1979), Tristan Tzara ou la spontanéité, in : Le Théâtre dada et surréaliste, Paris, Gallimard, p. 183-214.

CORVIN Michel (1971), Le théâtre Dada existe-t-il ? Tzara et RibemontDessaignes ou la problématique d'un théâtre dada, Revue d'histoire du théâtre 79 , p. $219-320$.

PAPACHRISTOS Katherine (1999), L'Inscription de l'oral et de l'écrit dans le théâtre de Tristan Tzara, Montréal, Peter Lang.

PaVIS Patrice (2004), Le Théâtre contemporain. Analyse de textes de Sarraute à Vinaver, Paris, Armand Colin.

SANOUILLET Michel (2005), Dada à Paris, Paris, Éditions du CNRS.

\footnotetext{
${ }^{22}$ Impression confiée à l'auditoire du colloque de l'UQÀM «Imaginer l'avant-garde aujourd'hui », le 7 juin 2010. La mise en scène évoquée faisait partie du Spectacle Dada, représenté le 21 et le 22 janvier 1967 au théâtre du Centre américain, à Paris, sous l'égide de l'Association pour l'Étude du Mouvement Dada, par le Théâtre de la Circonstance de Liège, direction Richard Tialans.
} 